\title{
Prognostic power of proadrenomedullin in community-acquired pneumonia is independent of aetiology
}

\author{
Salvador Bello*, Ana B. Lasierra*, Elisa Mincholé*, Sergio Fandos*, Maria \\ Angeles Ruiz", Elisabeth Vera*, Francisco de Pablo*, Miguel Ferrer ${ }^{+}$, \\ Rosario Menendez ${ }^{\S}$ and Antoni Torres ${ }^{+}$
}

ABSTRACT: Biomarkers are useful in community-acquired pneumonia (CAP). Recently, midregional (MR) proadrenomedullin (ProADM) has been shown to be of potential prognostic use. We sought to determine whether this prognostic role depends on the cause of CAP.

We conducted a prospective cohort study of immunocompetent patients with CAP. Pneumonia Severity Index (PSI) and CURB-65 score (confusion (abbreviated mental test score of $\leqslant 8$ ), urea $\geqslant 7 \mathrm{~mol} \cdot \mathrm{L}^{-1}$, respiratory rate $\geqslant 30$ breaths $\cdot \mathrm{min}^{-1}$, blood pressure $<90 \mathrm{mmHg}$ systolic or $<60 \mathrm{mmHg}$ diastolic, and age $\geqslant 65 \mathrm{yrs}$ ), blood C-reactive protein, procalcitonin, MR-proADM, and microbiological studies were systematically performed. Patients were grouped as bacterial, viral/atypical and mixed CAP, and were followed up at 30,90 and 180 days, and $1 \mathrm{yr}$.

We recruited 228 CAP patients. Identification of at least one pathogen was achieved in 155 (68\%) patients. MR-proADM levels closely correlated with increasing severity scores, and showed an important predictive power for complications and short- and long-term mortality (1 yr). Its addition to PSI and CURB-65 significantly improved their prognostic accuracy. A MR-proADM cutoff of $0.646 \mathrm{nmol} \cdot \mathrm{L}^{-1}$ identified $92 \%$ of patients scored as PSI classes IV and V as high risk. MRproADM outcome prediction power was not affected by different aetiologies.

MR-proADM has high short- and long-term prognostic accuracy, and increases the accuracy of clinical scores. The prognostic value of MR-proADM is not modified by different possible CAP aetiologies.

KEYWORDS: Aetiology, biomarkers, community-acquired pneumonia, midregional proadrenomedullin, outcome

ommunity-acquired pneumonia (CAP) is a disease with a very wide range of possible outcomes. Clinical severity scores, mainly the Pneumonia Severity Index (PSI) [1] and CURB65 score (confusion (abbreviated mental test score of $\leqslant 8$ ), urea $\geqslant 7 \mathrm{~mol} \cdot \mathrm{L}^{-1}$, respiratory rate $\geqslant 30$ breaths $\mathrm{min}^{-1}$, blood pressure $<90 \mathrm{mmHg}$ systolic or $<60 \mathrm{mmHg}$ diastolic, and age $\geqslant 65 \mathrm{yrs}$ ) [2], are useful tools for evaluating CAP-associated risk of mortality and, therefore, for making decisions about the most appropriate allocation of healthcare resources [2-5]. However, none of these tools is exempt from false-positive and -negative results. Biomarkers may improve the diagnostic accuracy of both PSI and CURB-65.

In recent years, many studies involving biomarkers for CAP have been performed [6]. C-reactive protein (CRP) and procalcitonin (PCT) have shown their usefulness for diagnosis [6], suspicion of typical bacteria as the causal agent [7], guiding antibiotic therapy [8], and assessing severity, mortality risk and the potential for complications $[9,10]$. Other new biomarkers have been proposed to provide information on severity and prognosis, including midregional (MR) proadrenomedullin (proADM).

MR-proADM is a fragment of proADM, the precursor of adrenomedullin (ADM), that is more stable than full-length proADM. MR-proADM is easy to measure and directly reflects levels of ADM. ADM is a member of the calcitonin peptide family that is widely expressed and intensively synthesised during severe infections, which has multiple effects, including immune
AFFILIATIONS

*Servicio de Neumología,

"Servicio de Bioquímica Clínica,

- Servicio de Microbiología, Hospital

Universitario Miguel Servet,

Zaragoza,

+Servicio de Neumología, Hospital Clínic i Provincial de Barcelona Institut d'Investigacions Biomèdiques August Pi i Sunyer, Universidad de Barcelona - Ciber de Enfermedades Respiratorias (Ciberes), Barcelona, and

${ }^{5}$ Servicio de Neumología, Hospital Universitario y Politécnico La Fe,

Ciberes, Valencia, Spain.

CORRESPONDENCE

S. Bello

Servicio de Neumología

Hospital Universitario Miguel Servet

Paseo Isabel La Católica 1-3

50009-Zaragoza

Spain

E-mail: sbello@salud.aragon.es

Received:

May 162011

Accepted after revision:

Sept 122011

First published online:

Nov 102011 
modulation, vasodilatation, antimicrobial activity and electrolyte balance [11, 12].

Four large, multicentre reports involving this biomarker in CAP outcomes have been published. They found a strong discriminatory power of MR-proADM for predicting serious complications [13], short-term [12, 14], mid-term [12] and longterm [15] mortality, and improved risk stratification of clinical scores [12-14]. These four multicentre studies included a very large number of patients, but some cohorts $[13,14]$ had been recruited for previous studies with heterogeneous objectives.

So far, no studies have been published that test whether the outcome prediction power of MR-proADM depends on the aetiology of CAP. Increased levels of PCT (and of CRP) have been found in Streptococcus pneumoniae CAP when compared with atypical bacterial or viral pneumonias $[7,16]$, but a possible aetiological discrimination capacity of MR-proADM is not yet known.

One objective of this study was to determine whether biomarkers, especially MR-proADM, alone or in association with the PSI and CURB-65 severity scores, are accurate in their assessment of outcome in hospitalised patients, independently of CAP aetiology. We also studied the potential role of biomarkers in predicting complications and short- and longterm mortality.

\section{MATERIAL AND METHODS \\ Selection of patients}

Consecutive adult ( $>18$ yrs of age) patients admitted by an emergency department (ED) physician to a 900-bed university hospital (Hospital Universitario Miguel Servet, Zaragoza, Spain) with a diagnosis of CAP were prospectively recruited within $24 \mathrm{~h}$ of their arrival. CAP was defined as an acute disease with a radiological infiltrate that was not previously present and not due to another known cause, and was associated with symptoms of lower respiratory tract infection. Exclusion criteria were: 1) severe immunodepression (HIV infection or severe haematological diseases); 2) immunosuppressive therapy (prednisone or equivalent dose of $>20 \mathrm{mg}$

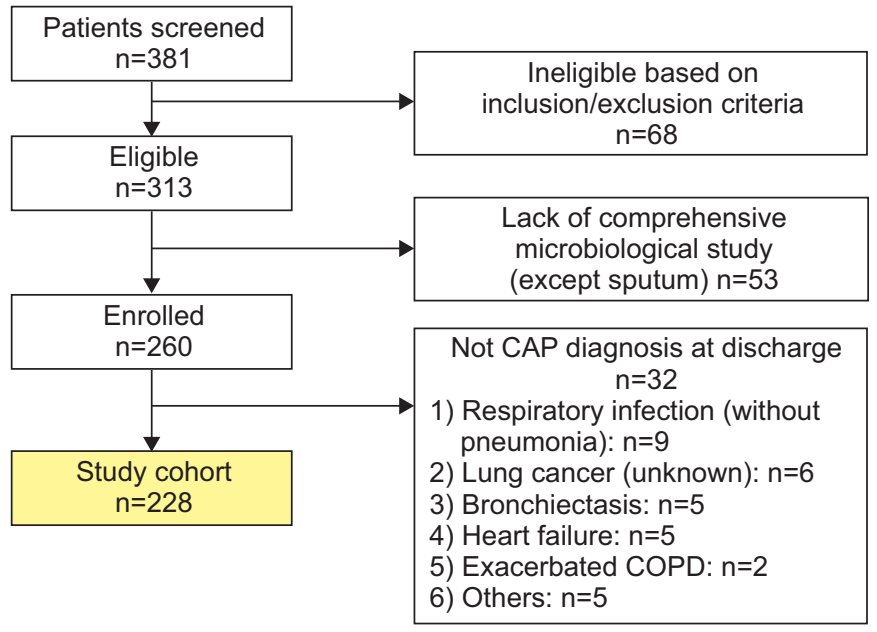

FIGURE 1. Flow diagram for patient enrolment or exclusion in the study. CAP community-acquired pneumonia; COPD: chronic obstructive pulmonary disease. daily for $>2$ weeks or any immunosuppressive regime therapy (azathioprine, cyclosporine, cyclophosphamide and/or other immunosuppressant drugs)); 3) leukopenia $(<1,000$ leukocytes per $\left.\mathrm{mm}^{3}\right)$ or neutropenia $\left(<500\right.$ neutrophils per $\left.\mathrm{mm}^{3}\right)$ and/or chemotherapy in the previous year; 4) pulmonary abscess (radiological cavitation), aspiration pneumonia and obstructive pneumonia; 5) possible nosocomial origin ( $<30$ days from hospital discharge); and 6) known active neoplasia. The study was approved by the Ethics Committee (Instituto Aragonés de Ciencias de la Salud, Zaragoza) and every patient gave informed consent. All patients were followed up during their hospital stay and those with a definitive diagnosis other than CAP were excluded (fig. 1).

During the study period, a sex- and age-matched control group was collected from subjects admitted to Orthopaedic Surgery, Digestive and Neurology Departments (Hospital Universitario Miguel Servet, Zaragoza, Spain). None of them had any suspicion of infectious or respiratory disease, immunosuppression, known neoplasia or recent trauma.

\section{Data collection}

The following variables were recorded: age; sex; anti-influenza vaccination $<1 \mathrm{yr}$ prior to the study; antipneumococcal vaccination $<5$ yrs prior to the study; tobacco consumption (nonsmoker, ex-smoker or current smoker, and pack-yrs of exposure in smokers); alcohol consumption (nondrinker, exdrinker or $<80$ or $>80 \mathrm{~g}$ alcohol per day in current drinkers); previous pneumonia; and previous recent (1 month) antibiotic treatment. In addition, at admission, the following were recorded: days of duration of disease; symptoms prior to infection; myalgia; CAP signs and symptoms; vital constants at the ED (respiratory and heart rates, arterial pressure and oxygen saturation); number of hours in the ED; arterial blood gas determinations (when performed); number of lobes involved and type of radiological condensation (alveolar, interstitial or mixed); and the presence of pneumothorax, atelectasis or bleeding.

PSI and CURB-65 scores were calculated for all patients. All patients were admitted to the hospital for $\geqslant 24 \mathrm{~h}$.

Adherence to Spanish antibiotic guidelines [17] and time of first dose after arrival at the hospital were also recorded. After discharge, patients were followed up and mortality was recorded at 30, 90 and 180 days, and 1 yr after inclusion in the study, when possible.

Data on CAP evolution and complications during hospitalisation were assessed, as were antibiotics (doses and treatment length, and first dose time), and days of admission, intensive care unit (ICU) admission, oxygen and mechanical ventilation (MV) requirements, and clinical stability. Complications included respiratory failure, need for MV, shock, left cardiac failure, renal failure, pleural effusion and others. Intrahospital mortality, causes of exitus and therapeutic failure were recorded. After 30 days, a clinical examination, blood analysis including a second serological sample, and chest radiography were performed.

Short-term mortality was defined as death due to any cause within the first 30 days. Mid- and long-term mortality were 


\begin{tabular}{|c|c|c|}
\hline TABLE 1 & \multicolumn{2}{|c|}{$\begin{array}{l}\text { Baseline characteristics of the } 228 \text { community- } \\
\text { acquired pneumonia patients on admission }\end{array}$} \\
\hline \multicolumn{2}{|l|}{ Age yrs } & $73(63-80)$ \\
\hline \multicolumn{2}{|l|}{ Age $\geqslant 65$ yrs } & $162(71.1)$ \\
\hline \multicolumn{2}{|l|}{ Males } & $139(61)$ \\
\hline \multicolumn{3}{|c|}{ Smoking status } \\
\hline \multicolumn{2}{|c|}{ Current smokers } & $55(24.1)$ \\
\hline \multicolumn{2}{|c|}{ Exposure pack-yrs } & $30(11-60)$ \\
\hline \multicolumn{2}{|l|}{ Ex-smoker } & $89(39.0)$ \\
\hline \multicolumn{2}{|c|}{ Exposure pack-yrs } & $48(30-75)$ \\
\hline \multicolumn{3}{|c|}{ Coexisting illnesses } \\
\hline \multicolumn{2}{|c|}{ Neoplastic disease, not active } & $30(13.2)$ \\
\hline \multicolumn{2}{|c|}{ Heart disease } & $84(36.8)$ \\
\hline \multicolumn{2}{|c|}{ Cerebrovascular disease } & $47(20.6)$ \\
\hline \multicolumn{2}{|l|}{ COPD } & $72(31.6)$ \\
\hline \multicolumn{2}{|c|}{ Liver disease } & $7(3.1)$ \\
\hline \multicolumn{2}{|c|}{ Renal disease } & $35(15.4)$ \\
\hline \multicolumn{2}{|c|}{ Chronic renal disease } & $27(11.8)$ \\
\hline \multicolumn{2}{|c|}{ Diabetes mellitus } & $44(19.3)$ \\
\hline \multicolumn{2}{|c|}{ Bronchiectasis } & $11(4.8)$ \\
\hline \multicolumn{3}{|c|}{ Clinical findings } \\
\hline \multicolumn{2}{|c|}{ Antibiotic pre-treatment } & $64(28.1)$ \\
\hline \multicolumn{2}{|c|}{ Pre-enrolment duration of symptoms days } & $3.5(2-6)$ \\
\hline Altered mer & tal status & $27(11.8)$ \\
\hline Pulse $>125$ & beats $\cdot \min ^{-1}$ & $20(8.8)$ \\
\hline Respiratory & rate $\geqslant 30$ breaths $\cdot \mathrm{min}^{-1}$ & $43(18.9)$ \\
\hline Systolic BP & $<90 \mathrm{mmHg}$ & $26(11.4)$ \\
\hline Diastolic BF & $<60 \mathrm{mmHg}$ & $46(20.2)$ \\
\hline Body tempe & rature $<35$ or $>40^{\circ} \mathrm{C}$ & $3(1.3)$ \\
\hline Radiographi & findings & \\
\hline Unilobar rac & liograph & $190(83.3)$ \\
\hline Multilobar/b & lateral radiograph & $38(16.7)$ \\
\hline Pleural effu & & $53(23.2)$ \\
\hline PSI & & \\
\hline Score & & $102.9 \pm 40.0$ \\
\hline Class I & & $19(8.3)$ \\
\hline Class II & & 30 (13.2) \\
\hline Class III & & $40(17.5)$ \\
\hline Class IV & & 85 (37.3) \\
\hline Class V & & $54(23.7)$ \\
\hline CURB-65 scc & & \\
\hline 0 & & $33(14.5)$ \\
\hline 1 & & 60 (26.3) \\
\hline 2 & & $86(37.7)$ \\
\hline 3 & & $32(14.0)$ \\
\hline 4 & & $12(5.3)$ \\
\hline 5 & & $5(2.2)$ \\
\hline Aetiology & & \\
\hline Typical bac & erial & $57(25.0)$ \\
\hline Viral/atypica & bacterial & $57(25.0)$ \\
\hline Mixed bact & rial plus viral & $41(18.0)$ \\
\hline Unknown & & 73 (32.0) \\
\hline
\end{tabular}

Data are presented as median (interquartile range), $\mathrm{n}(\%)$ or mean \pm SD. COPD: chronic obstructive pulmonary disease; BP: blood pressure; PSI: Pneumonia Severity Index; CURB-65: confusion (abbreviated mental test score of $\leqslant 8$ ), urea $\geqslant 7 \mathrm{~mol} \cdot \mathrm{L}^{-1}$, respiratory rate $\geqslant 30$ breaths $\cdot \mathrm{min}^{-1}$, blood pressure $<90 \mathrm{mmHg}$ systolic or $<60 \mathrm{mmHg}$ diastolic, and age $\geqslant 65 \mathrm{yrs}$. defined as death due to any cause up to 180 days and $1 \mathrm{yr}$, respectively, after inclusion in the study.

\section{Sample collection and processing}

Determination of leukocyte count, CRP, PCT and MR-proADM

Venous blood samples were collected from CAP patients and controls on admission to the ED, within $6 \mathrm{~h}$ of arrival. Serum CRP was measured by immunoturbidimetry using the highly sensitive near-infrared particle immunoassay methodology (IMMAGE 800; Beckman Coulter, San Diego, CA, USA). The assay has an analytical detection limit of $0.06 \mathrm{mg} \cdot \mathrm{L}^{-1}$ and a functional assay sensitivity of $0.11 \mathrm{mg} \cdot \mathrm{L}^{-1}$. $\mathrm{PCT}$ and MRproADM concentrations were determined using sandwich immunoassays and time-resolved amplified cryptate emission measurement (PCT Sensitive KRYPTOR and MR-proADM KRYPTOR; BRAHMS, Hennigsdorf, Germany) as described in detail previously [18]. The analytical detection limits and functional assay sensitivities for the assays were 0.02 and $0.06 \mathrm{ng} \cdot \mathrm{mL}^{-1}$ for $\mathrm{PCT}$, and 0.05 and $0.25 \mathrm{nmol} \cdot \mathrm{L}^{-1}$ for $\mathrm{MR}-$ proADM, respectively. Measurements of PCT and MRproADM were performed in our laboratory (Servicio de Bioquímica Clínica, Hospital Universitario Miguel Servet) in a blinded fashion without knowing the clinical parameters and microbiological results.

\section{Microbiological tests}

Blood was drawn from CAP patients in the ED shortly after CAP diagnosis. Sputum was obtained when possible, before antibiotics were administered, and immediately sent for Gram staining and culture, and only samples containing a preponderance of leukocytes and a few squamous epithelial cells were considered acceptable. Another blood sample was obtained for the first serological Mycoplasma pneumoniae (complement fixation test; Virion Serion Institut, Wurzburg, Germany) and Chlamydophila pneumoniae (ELISA; Savyon Diagnostics Ltd, St Ashdod, Israel) tests. The second serological tests were performed on blood obtained during the 30-day follow-up visit. Urine was collected during the first $24 \mathrm{~h}$ and tested for $S$. pneumoniae and Legionella pneumophila antigens (BINAX Now; Binax, Portland, ME, USA).

Nasopharyngeal aspirates were obtained and processed for viral antigens using the direct fluorescence antibody (DFA) assay and two different viral PCR tests. DFA was performed for influenza virus A and B, parainfluenza virus 1, 2 and 3, adenovirus (AdV), human metapneumovirus (hMPV), and respiratory syncytial virus (RSV) (D3 Double Duet DFA Respiratory Virus Screening and ID Kit; Diagnostic Hybrids, Athens, $\mathrm{OH}, \mathrm{USA}$ ). The first PCR was a multiplex reverse transcriptase (RT) nested PCR assay for 14 respiratory viruses (influenza virus A, B and C, RSV A and B, AdV, coronavirus 229E and OC43, enterovirus, parainfluenza virus 1, 2, 3 and 4, and rhinovirus) [19]; the second test was a commercial RT-PCR kit, the ResPlex II Plus Panel (Qiagen LiquiChip System; Qiagen $\mathrm{GmbH}$, Hamburg, Germany) [20] for detection of 18 viruses (influenza virus A and B, RSV A and B, parainfluenza virus 1, 2, 3 and 4, hMPV A and B, enterovirus (coxsackievirus/echovirus), rhinovirus, AdV B and E, coronavirus NL63, HKU1, 229E and OC43, and bocavirus). Nucleic acids had been extracted from nasopharyngeal aspirates immediately after their reception. 

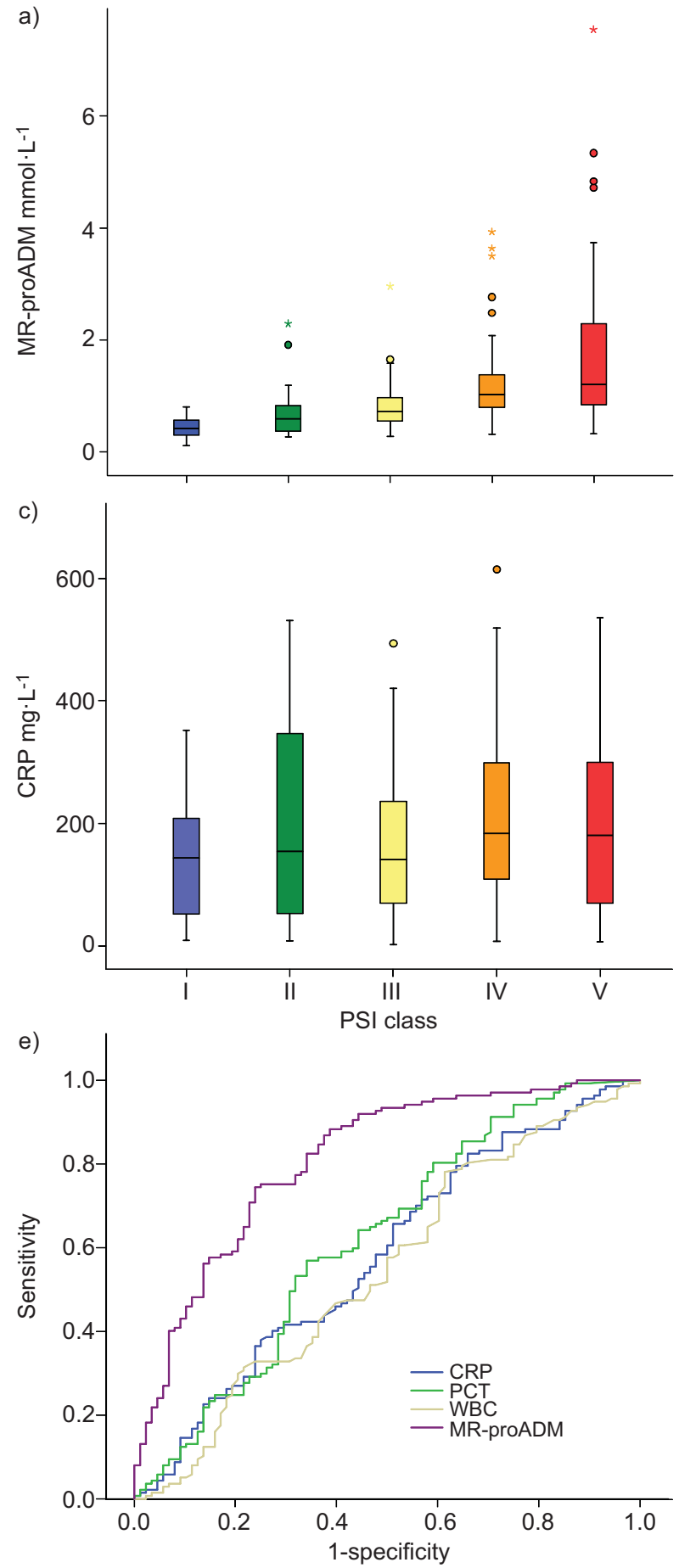
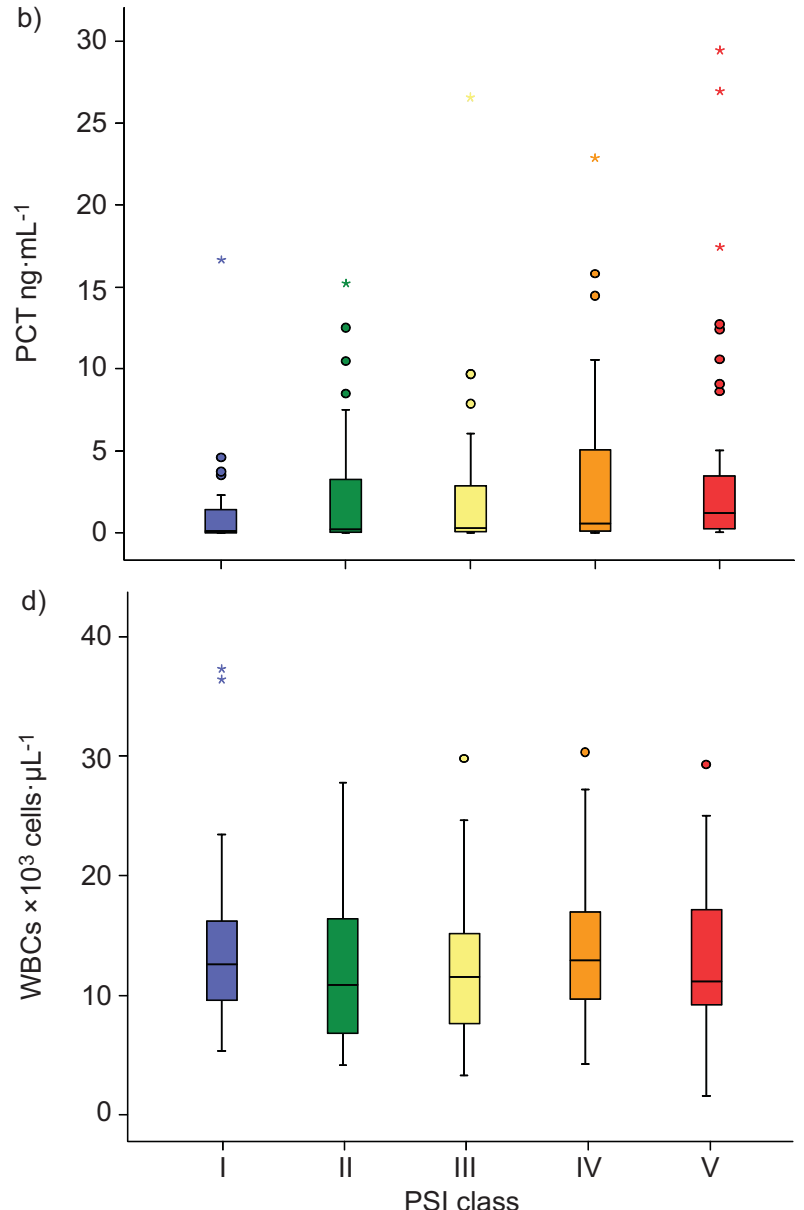

FIGURE 2. Biomarkers and Pneumonia Severity Index (PSI) severity score. Distribution of a) midregional (MR) proadrenomedullin (proADM), b) procalcitonin (PCT), c) Creactive protein (CRP) and d) white blood cells (WBCs) by PSI classes. MR-proADM was the only biomarker able to distinguish between all different risk classes of PSI. When patients were classified into low- (I-III) and high-risk (IV-V) PSI classes, median levels of MR-proADM ( $p<0.0001)$, PCT $(p=0.002)$ and CRP ( $p=0.025)$ were significantly higher in high- (IV-V) than in low-risk classes (I-III). Analogous results were not found for WBCs $(p=0.183)$. e) Receiver operating characteristic analysis to assess the power of these biomarkers in order to discriminate between low- (PSI I-III) and high-risk (PSI IV-V) community-acquired pneumonia (CAP), showed an MR-proADM area under the curve (AUC) $(0.811,95 \% \mathrm{Cl} 0.753-0.869)$ that was significantly higher than the AUCs of PCT $(0.620,95 \% \mathrm{Cl} 0.542-0.697)$ and $\mathrm{CRP}(0.588,95 \% \mathrm{Cl} 0.511-0.665)(\mathrm{p}<0.0001$ and $p<0.0001$, respectively). Therefore, MR-proADM presented the best AUC and may be considered a good predictor of CAP severity.

Patients with CAP were divided into three groups: bacterial, viral/atypical and mixed. S. pneumoniae and other Gram-positive and -negative bacteria classically considered producers of CAP, as well as L. pneumophila, were included as "bacterial". M. pneumoniae and C. pneumoniae were considered "atypical" and added to the viral group $[7,16]$. One or more typical bacteria 


\begin{tabular}{lc} 
TABLE 2 30-day complications & \\
\hline 30-day complication & Patients $\mathbf{n}$ \\
\hline & \\
Respiratory failure without hypercapnia & 81 \\
Respiratory acidosis & 11 \\
MV & 8 \\
ICU admission & 7 \\
Nosocomial pneumonia & 1 \\
Pleural effusion & 30 \\
Empyema & 2 \\
Pneumothorax & 2 \\
Death & 13 \\
Left cardiac failure & 37 \\
New hyperglycaemia/diabetes mellitus decompensation & 31 \\
Acute renal failure & 13 \\
Tract urinary infection & 15 \\
Tachyarrythmias & 12 \\
Septic shock & 11 \\
Confusional syndrome & 12 \\
Acute gastroenteritis & 7 \\
Coronary acute syndrome & 12 \\
Cardiogenic shock & 4 \\
Cerebellum infarction & 110 \\
Complete AV blockade & \\
Pericardial effusion & 1 \\
Endocarditis & 1 \\
Hypertensive crisis & 1 \\
LLE cellulitis & 1 \\
Coagulopathy & 1 \\
Fournier gangrene & 1 \\
Right arterial iliofemoral obstruction & 1 \\
Right psoas haematoma & 1 \\
Rectorrhagia & 1 \\
Total complications & 1 \\
\hline & 1 \\
\hline
\end{tabular}

Numbers of patients who suffered each type of complication of a total of 146 patients with complications are shown. MV: mechanical ventilation; ICU: intensive care unit; AV: atrioventricular; LLE: left lower extremity.

(and L. pneumophila) in association with one or more viruses or atypical bacteria were considered "mixed" CAP.

\section{Statistical analysis}

Baseline and follow-up characteristic data are presented as n (\%) for categorical variables, and as mean \pm SD or median (interquartile range (IQR)) for continuous variables, as appropriate.

Frequency comparison was performed by Chi-squared test or Fisher's exact test, when appropriate, for qualitative variables. Correlations were analysed using the Spearman rho correlation analysis. Two-group comparisons were performed using the Wilcoxon/Mann-Whitney U-test and multigroup comparisons using the Kruskal-Wallis test.

We constructed receiver operating characteristic (ROC) curves and determined the area under the curve (AUC). The AUC and its $95 \%$ confidence intervals were estimated for each biomarker and compared using a nonparametric method. Sensitivity, specificity, positive and negative predictive values, and

\begin{tabular}{|c|c|c|c|c|}
\hline TABLE 3 & $\begin{array}{l}\text { Jnivariate and } n \\
\text { analyses }\end{array}$ & Itivariate & ogistic regress & ion \\
\hline \multirow[t]{2}{*}{ Variable } & \multicolumn{2}{|c|}{ Univariate } & \multicolumn{2}{|c|}{ Multivariate } \\
\hline & OR (95\% Cl) & p-value & OR $(95 \% \mathrm{Cl})$ & p-value \\
\hline Aetiology & & 0.059 & & \\
\hline Bacterial & $1.35(0.65-2.80)$ & 0.423 & & \\
\hline Viral & $0.804(0.39-1.64)$ & 0.547 & & \\
\hline Mixed & $2.93(1.14-7.53)$ & 0.025 & & \\
\hline PSI & $2.08(1.61-2.69)$ & 0.000 & & \\
\hline CURB-65 & 1.99 (1.49-2.69) & 0.000 & $1.60(1.15-2.22)$ & 0.005 \\
\hline MR-proADM & $3.51(1.86-6.63)$ & 0.000 & $2.24(1.18-4.24)$ & 0.014 \\
\hline CRP & $1.04(1.01-1.06)$ & 0.002 & & \\
\hline РСТ & $1.07(0.99-1.14)$ & 0.058 & & \\
\hline WBCs & $1.01(0.96-1.05)$ & 0.837 & & \\
\hline AUC $(95 \% \mathrm{Cl})$ & & & $0.729(0.659-$ & $0.799)$ \\
\hline
\end{tabular}

PSI: Pneumonia Severity Index; CURB-65: confusion (abbreviated mental test score of $\leqslant 8$ ), urea $\geqslant 7 \mathrm{~mol} \cdot \mathrm{L}^{-1}$, respiratory rate $\geqslant 30$ breaths $\cdot \mathrm{min}^{-1}$, blood pressure $<90 \mathrm{mmHg}$ systolic or $<60 \mathrm{mmHg}$ diastolic, and age $\geqslant 65 \mathrm{yrs}$; MR: midregional; proADM: proadrenomedullin; CRP: C-reactive protein; $\mathrm{PCT}$ : procalcitonin; WBC: white blood cell; AUC: area under the curve. Dependent variable: complications. Bold indicates statistically significant $p$-values.

positive- and negative-likelihood ratios (LHR+ and LHR-, respectively) were calculated for each cut-off value of the biomarkers.

Bonferroni correction of critical p-values was applied when performing a high number of comparisons. In order to avoid a lot of spurious positives, the alpha value was lowered to account for the number of comparisons being performed.

Logistic regression was used to analyse the effect of adding biomarkers to the PSI and CURB-65 score for short-, mid- and long-term mortality. The accuracy of these models at predicting mortality was compared with the accuracy of the PSI or CURB-65 score by comparing their AUCs.

We used two different tests to determine whether there was an association between different aetiologies and MR-proADM. First, we performed a nonparametric test to evaluate the association between each group of the categorical variable (aetiology) and the quantitative variable (MR-proADM) by means of the Mann-Whitney U-test. We also transformed the MR-proADM levels into a categorical variable (MR-proADM quartiles) and performed a comparison with aetiology using the Chi-squared test.

All tests were two-tailed and p-values $<0.05$ were considered to indicate statistical significance, applying Bonferroni correction when appropriate. All statistical analyses were performed using SPSS version 15.0 (SPSS Inc., Chicago, IL, USA), except the comparisons of the AUCs, which were carried out using MedCalc version 11.4.2.0 (MedCalc Software, Mariakerke, Belgium).

\section{RESULTS}

\section{Patients and controls}

260 patients with a diagnosis of CAP were included in the study. Of these patients, $32(12.3 \%)$ were excluded because 
they had an alternative diagnosis. A total of 228 subjects constituted the study cohort, with a median age of 73 yrs (IQR 63-80 yrs) (table 1).

Each patient received antibiotic therapy according to Spanish Respiratory Society Guidelines [17] and an initial dose was administered within $6 \mathrm{~h}$ of arriving in the ED in $192(84.2 \%)$ subjects with CAP. All biomarkers were available on admission in 225 (99\%) subjects. $139(60.96 \%)$ patients were identified as being at high risk by PSI (classes IV and V) and 135 (59.2\%) by CURB-65 score $(\geqslant 2)$.

$13(5.8 \%)$ out of 224 CAP patients followed up for 30 days after admission died. The cause of death of 12 of these patients was the initial CAP or its complications and one patient died due to cardiogenic shock. A further seven patients had died at 90 days (20 out 220 patients, 9.1\%) and their causes of death were: Alzheimer's disease (two patients), dilated cardiomyopathy (one patient), new CAP (one patient), complications of initial CAP (one patient) and unknown causes (two patients). Six more had died at 180 days (26 out of $218,11.9 \%$ ), because of chronic obstructive pulmonary disease (COPD) (two patients), ischaemic heart disease, pulmonary fibrosis, new CAP and vascular dementia (one patient each). At 1 yr of follow-up, four more patients had died (18 out 150 patients, 12\%), and their causes of death included new CAP (two patients), renal failure and Alzheimer's disease (one patient), and small bowel neoplasm (one patient). Excluding 30-day mortality, all but one patient (at 90 days) died due to causes other than the initial CAP. Thus, the number of patients studied at each timepoint was 228 patients at admission, 224 at 30 days, 220 at 90 days, 218 at 180 days and 150 patients at 1 yr.

The control group was composed of 70 age- and sex-matched subjects. Comorbidities in this group were similar to those of CAP patients. Nasopharyngeal aspirates were obtained in 60 controls for viral search, including both PCR tests. We did not obtain paired blood samples for serological studies in these subjects.

\section{Biomarker levels and CAP severity}

Levels of MR-proADM on admission increased significantly with increasing severity of CAP according to the PSI $(p<0.0001)$ and CURB-65 scores $(p<0.0001)$. In terms of severity assessment, correlation coefficients (CCs) between MR-proADM and PSI and CURB-65 scores were higher (CC 0.562 and 0.561 , respectively) than those of PCT (CC 0.228 and 0.283 ), and there was a good correlation between both clinical scores (CC 0.718). MR-proADM was the only biomarker able to distinguish between all different PSI score risk classes $(p<0.05)$ (fig. 2a-d). ROC analysis to discriminate between low-risk (PSI I-III) and high-risk (PSI IV-V) CAP showed that MR-proADM was the best biomarker (fig. 2e).

The optimal cut-off for MR-proADM was $0.646 \mathrm{nmol} \cdot \mathrm{L}^{-1}$, with a sensitivity of $92.1 \%$, specificity of $55.1 \%$, positive predictive value of $76.2 \%$ and negative predictive value of $80.3 \%$. With this cut-off, of 139 patients classified by PSI score as high-risk (classes IV and V), 128 (92\%) would have been correctly classified by MR-proADM levels, whereas only 11, according to PSI score, would have been "wrongly" considered as low-risk CAP (PSI I-III). 10 of these 11 patients were elderly (68-95 yrs of age); seven of them had a CURB-65 score of 1, one had a score of 2 and three had a score of 3 . All the patients had comorbidities. Seven patients developed complications, but radiological resolution was achieved in all 11 and they are all still alive.

\section{Complications and mortality}

Complications in CAP patients

$146(64 \%)$ patients suffered 310 significant complications within 30 days of admission (table 2). We found significantly

TABLE 4 Levels of biomarkers according to short-, mid- and long-term mortality

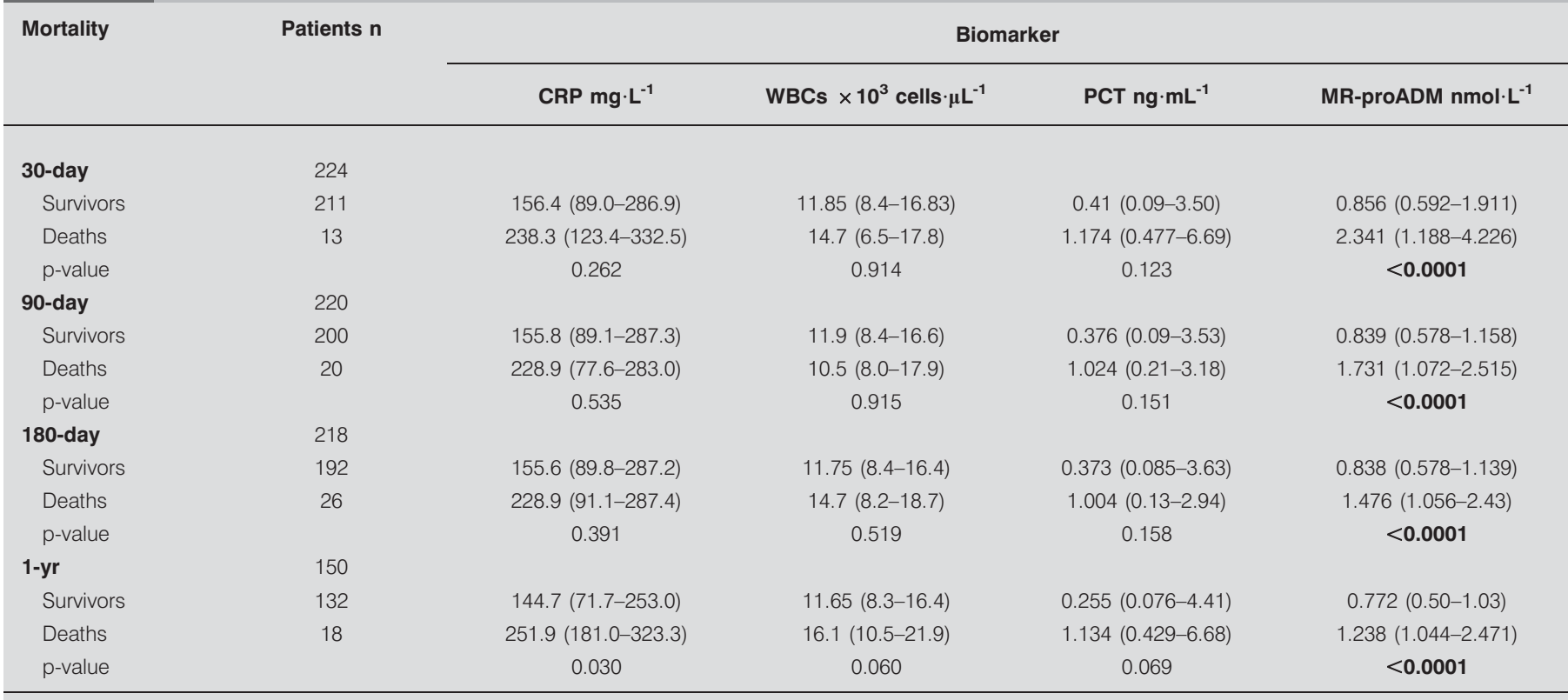

Data are presented as median (interquartile range), unless otherwise stated. CRP: C-reactive protein; WBC: white blood cell; PCT: procalcitonin; MR: midregional; proADM: proadrenomedullin. Bonferroni correction was applied $(p \leqslant 0.003125)$. Bold indicates statistically significant $p$-values. 


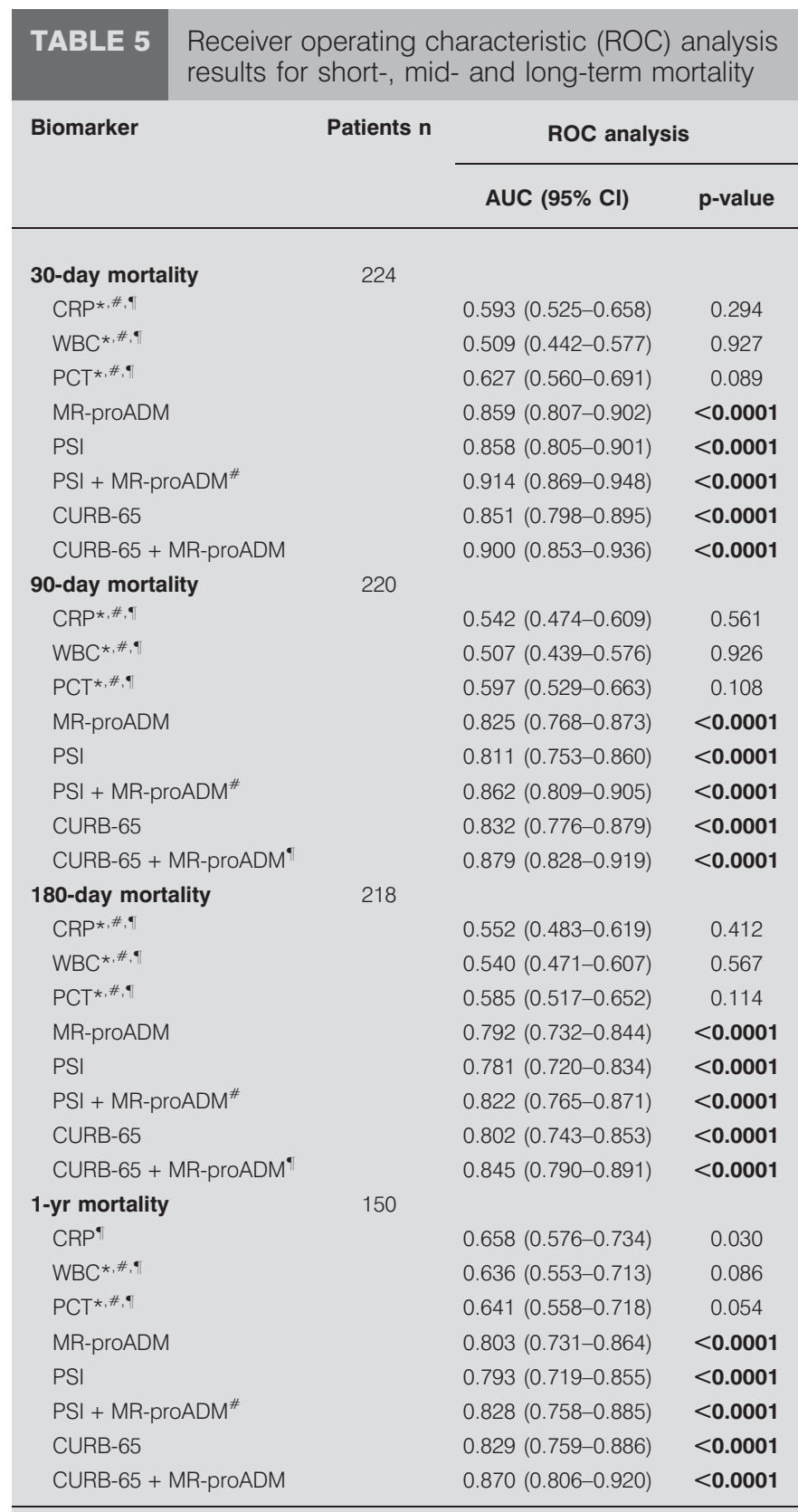

AUC: area under the curve; CRP: C-reactive protein; WBC: white blood cells; PCT, procalcitonin; MR: midregional; proADM: proadrenomedullin; PSI: Pneumonia Severity Index; CURB-65: confusion (abbreviated mental test score of $\leqslant 8$ ), urea $\geqslant 7 \mathrm{~mol} \cdot \mathrm{L}^{-1}$, respiratory rate $\geqslant 30$ breaths $\cdot \mathrm{min}^{-1}$, blood pressure $<90 \mathrm{mmHg}$ systolic or $<60 \mathrm{mmHg}$ diastolic, and age $\geqslant 65 \mathrm{yrs}$; PSI + MRproADM: logistic regression model combining MR-proADM levels with PSI risk score; CURB-65 + MR-proADM: logistic regression model combining MRproADM levels with CURB-65 risk score. Bonferroni correction was applied $(p \leqslant 0.00156)$. Bold indicates statistically significant $p$-values. ${ }^{*}: p<0.05$ for comparison of AUCs with that of MR-proADM; ${ }^{*}: p<0.05$ for comparison of AUCs with that of PSI; ${ }^{\circ}: p<0.05$ for comparison of AUCs with that of CURB-65.

higher levels of MR-proADM $(p<0.0001)$, PCT $(p=0.001)$ and CRP $(p=0.004)$, and higher PSI $(p<0.0001)$ and CURB-65 $(\mathrm{p}<0.0001)$ scores in patients with complications.
In the ROC analysis, the AUCs were as follows. PSI: 0.729 (95\% CI, 0.659-0.800); MR-proADM: 0.706 (95\% CI, 0.636-0.776); CURB-65: 0.693 (95\% CI, 0.619-0.767); PCT: 0.635 (95\% CI, 0.557-0.714); CRP: 0.618 (95\% CI, 0.542-0.694). No significant differences were found when the AUCs for PSI and MRproADM were compared $(\mathrm{p}=0.547)$.

The optimal cut-off for predicting complications for MRproADM was $0.833 \mathrm{nmol} \cdot \mathrm{L}^{-1}$ (sensitivity $67.35 \%$; specificity $66.23 \%$; LHR+ 1.99; LHR- 0.49). The multivariate analysis selected only CURB-65 and MR-proADM as predictors of complications (table 3).

\section{Short-, mid- and long-term mortality}

Table 4 shows that MR-proADM was the only biomarker able to discriminate between patients who died at 30, 90 and 180 days, and $1 \mathrm{yr}(\mathrm{p}<0.0001)$. ROC analysis showed that the AUC for MR-proADM was significantly higher than those for PCT, CRP and WBC, and similar to those for the PSI and CURB-65 scores (table 5). Logistic regression models combining MR-proADM levels with either PSI or CURB-65 risk scores showed significant effects $(p<0.05)$.

The optimal cut-off for predicting 30-day mortality for MR-proADM was $1.066 \mathrm{nmol} \cdot \mathrm{L}^{-\mathbf{1}}$, with sensitivity of $92.3 \%$, specificity of $67.46 \%$, LHR+ of 2.84 and LHR- of 0.11 . Only the AUC of model combining MR-proADM with PSI differed from the AUC of PSI alone $(p=0.0002)$. For $90-$ and 180-day mortality, the combination of MR-proADM levels with either PSI or CURB-65 scores yielded significant effects $(\mathrm{p}<0.05)$.

For 1-yr mortality, only a model combining MR-proADM and PSI discriminated mortality better than the PSI score alone $(p=0.0392)$. Logistic regression models combining CRP with clinical scores or MR-proADM levels did not yield significant effects (table 5 and fig. 3).

The multivariate analysis of mortality at 30 and 180 days selected CURB-65 and MR-proADM as predictors (table 6).

\section{Aetiology}

The causative pathogen was found in 155 (68\%) patients: bacterial infection in $57(25.0 \%)$ patients, viral/atypical bacterial infection in $57(25.0 \%)$ and mixed (bacterial plus virus/atypical) infection in 41 (17.98\%) (table 7). All 60 controls with viral study had a negative DFA assay, and genomes of $\mathrm{AdV}$ and parainfluenza virus 4 were found in three and one subject, respectively (four out of 60, 6.7\%).

\section{Biomarker levels and aetiology}

Levels of biomarkers in CAP patients with different aetiologies are shown in table 8. PCT was the only biomarker that showed significant differences $(\mathrm{p}<0.0001)$ between bacterial (median $2.402 \mathrm{ng} \cdot \mathrm{mL}^{-1}$ ) and viral/atypical CAP (median $0.272 \mathrm{ng} \cdot \mathrm{mL}^{-1}$ ). PCT was also the only biomarker that distinguished viral/ atypical from mixed pneumonia (median $1.568 \mathrm{ng} \cdot \mathrm{mL}^{-1}$; $p=0.007)$. None of the biomarkers discriminated between bacterial and mixed aetiology.

We did not find any association between aetiology and MRproADM ( $\mathrm{p}>0.05)$. Table 8 shows that MR-proADM levels were similar in all three aetiological groups. 

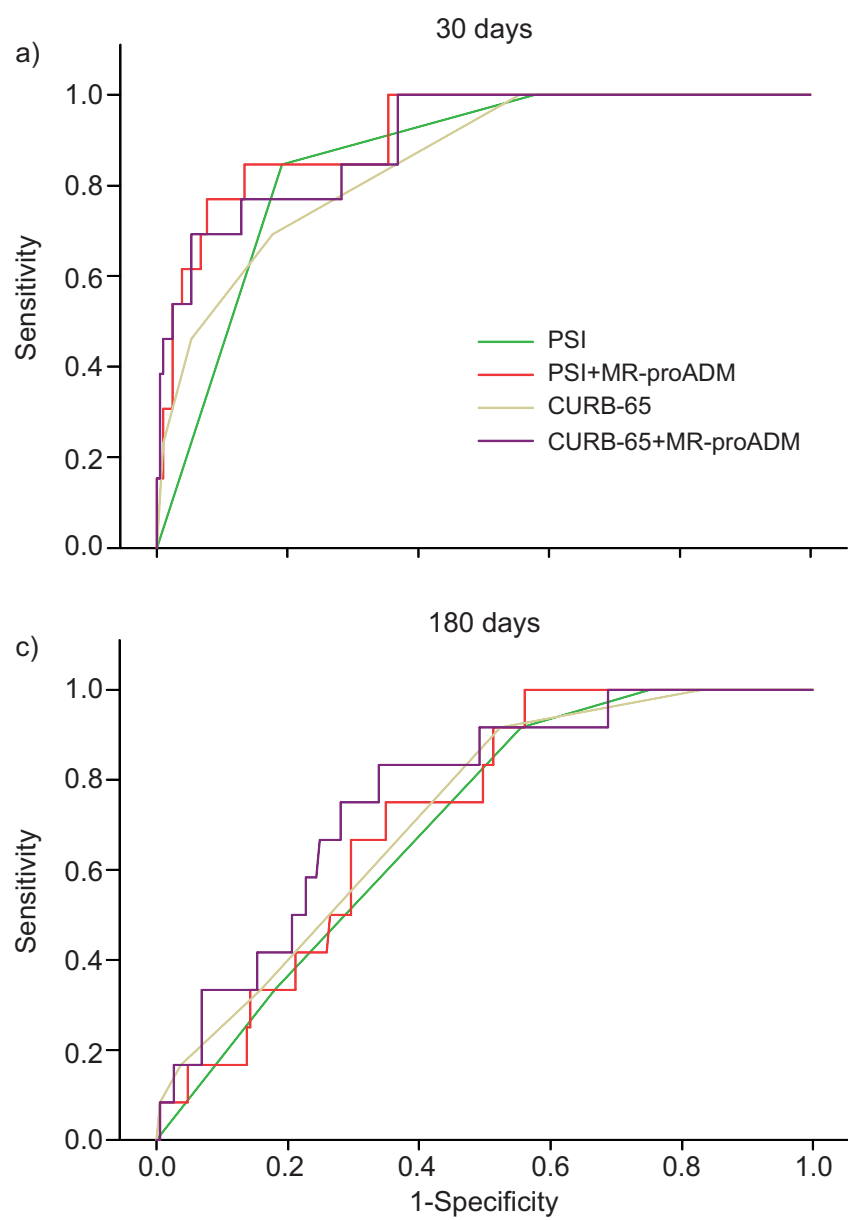

b)

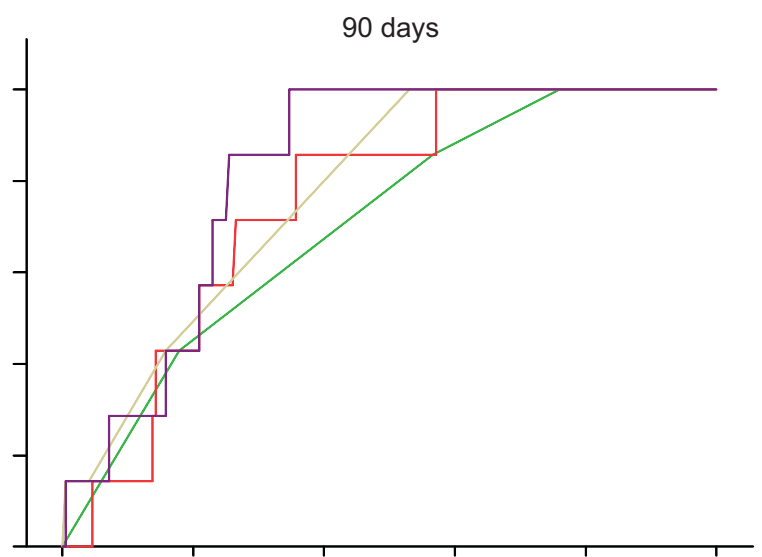

d)

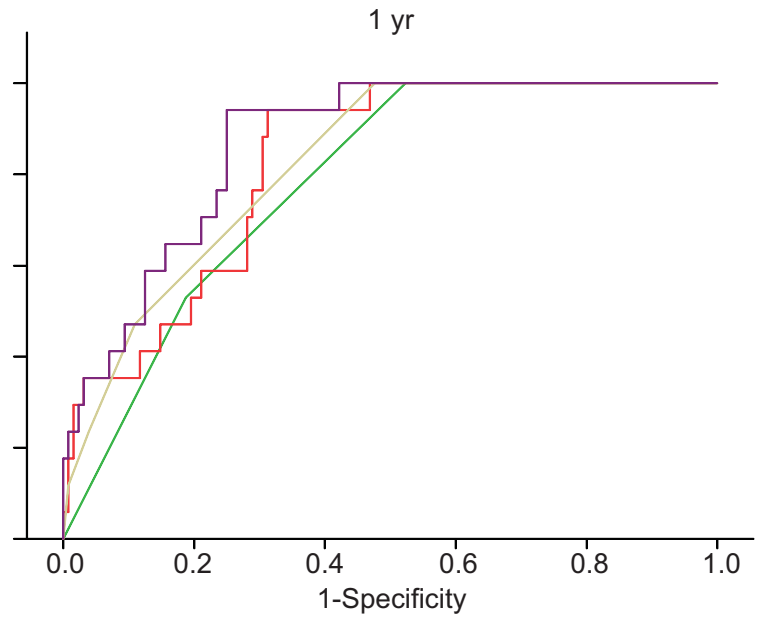

FIGURE 3. Receiver operating characteristic curves for short-, mid- and long-term mortality comparing Pneumonia Severity Index (PSI)/CURB-65 score (confusion (abbreviated mental test score of $\leqslant 8$ ), urea $\geqslant 7 \mathrm{~mol} \cdot \mathrm{L}^{-1}$, respiratory rate $\geqslant 30$ breaths $\cdot \mathrm{min}^{-1}$, blood pressure $<90 \mathrm{mmHg}$ systolic or $<60 \mathrm{mmHg}$ diastolic, and age $\geqslant 65 \mathrm{yrs}$ ) with the corresponding logistic regression models (PSI/CURB-65 score plus midregional (MR) proadrenomedullin (proADM)). a) Mortality at 30 days: combining MR-proADM with PSI significantly improved the prognostic accuracy of PSI alone (area under the curve (AUC) 0.858), with an AUC of 0.914 ( $p=0.0002$ ). However, the AUC of the model combining MR-proADM and CURB-65 (0.900), although higher, did not differ from the AUC of CURB-65 alone (0.851; $p=0.097)$. Mortality at b) 90 and c) 180 days: combination of MR-proADM levels with either PSI or CURB-65 risk score improved the prognostic accuracy over that of the PSI and CURB-65 scores alone ( $p<0.05)$. $d$ ) Mortality at $1 \mathrm{yr}$ : combining MR-proADM levels with PSI risk score (AUC 0.828) better discriminated mortality at $1 \mathrm{yr}$ than PSI score alone (0.793; $p=0.0392$ ). However, the AUC of the model combining MR-proADM and CURB-65 (0.870,95\% Cl 0.806-0.920), although higher, did not differ from the AUC of CURB-65 alone $(0.829 ; \mathrm{p}=0.089)$.

\section{DISCUSSION}

Our findings confirm that initial levels of MR-proADM in CAP patients have considerable predictive power for complications, and short-, mid- and long-term mortality of CAP. This predictive power of mortality was independent of the aetiology of CAP.

In our study, MR-proADM was the only biomarker able to distinguish between every PSI class $(p<0.05)$, whereas PCT only discriminated the extreme levels of severity (class I-III from IV-V). MR-proADM was the best biomarker (AUC of 0.811 ), with an optimal cut-off of $0.646 \mathrm{nmol} \cdot \mathrm{L}^{-1}$. With this cutoff, 128 (92\%) out of 139 patients in PSI classes IV and V would have been correctly classified. This cut-off could be used in the ED, in association with clinical severity scores, when deciding whether to admit patients with CAP to the hospital or manage them as outpatients. Previous studies found a good correlation between MR-proADM levels and PSI [11, 14] and CRB-65 (confusion (abbreviated mental test score of $\leqslant 8$ ), respiratory rate $\geqslant 30$ breaths $\cdot \mathrm{min}^{-1}$, blood pressure $<90 \mathrm{mmHg}$ systolic or
$<60 \mathrm{mmHg}$ diastolic, and age $\geqslant 65 \mathrm{yrs}$ ) [12] severity scores, whereas another study concluded that PCT can identify lowrisk patients across CRB-65 classes [10].

$146(64 \%)$ of our patients suffered 310 significant complications within 30 days of admission, and showed significantly higher levels of MR-proADM, PCT and CRP, and significantly higher PSI and CURB-65 scores, although the best AUCs were those for PSI, MR-proADM and CURB-65; these AUCs were better than those for PCT and CRP. SCHUETZ et al. [13] found that MR-proADM had a greater discriminatory power than PSI and CURB-65 scores. These findings may be of use when deciding where to manage CAP, especially in subjects with low-range severity scores and high levels of biomarkers. The decision to treat these cases as outpatients should probably be reconsidered [13].

MR-proADM has shown to be an accurate biomarker for shortterm mortality in CAP [11-14] and sepsis [21, 22]. In our study, the addition of MR-proADM levels to the PSI clinical severity score significantly improved 30-day prognostic accuracy. 


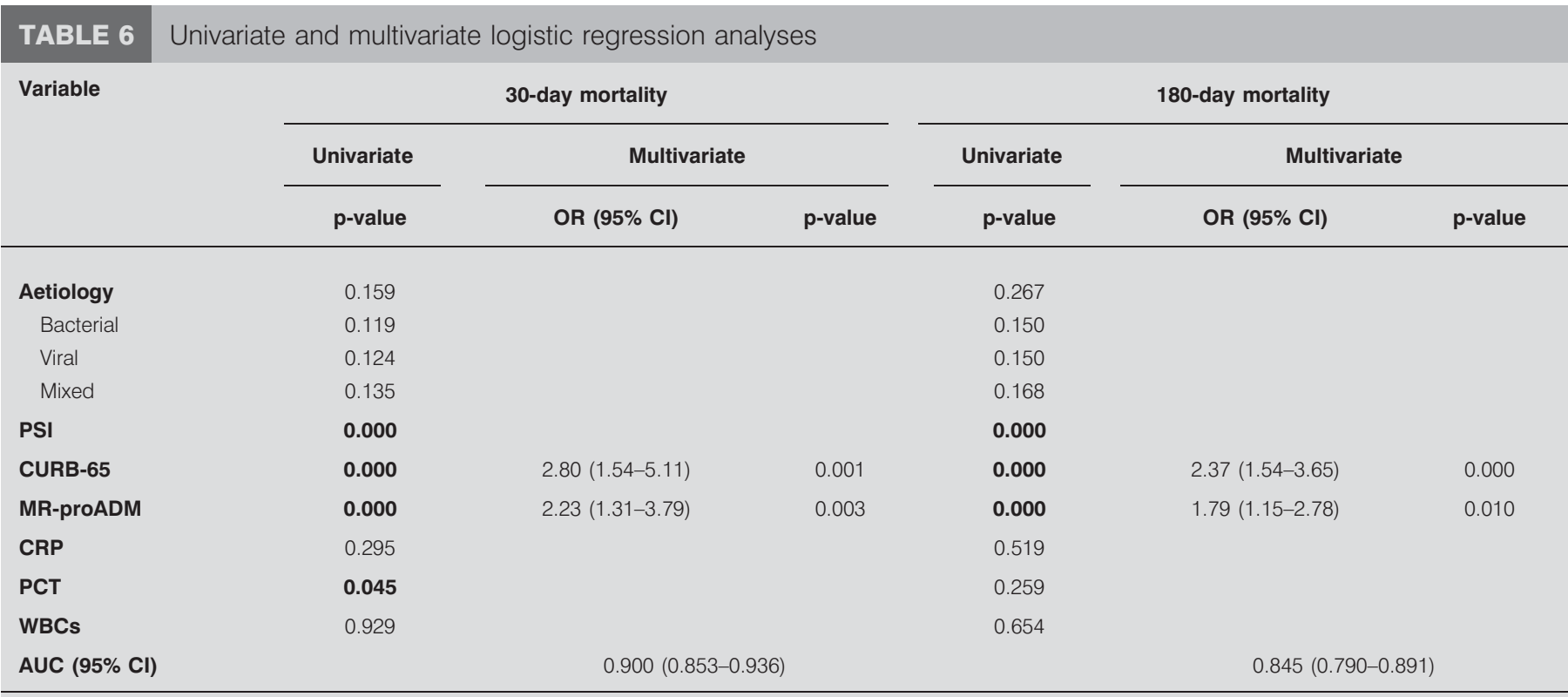

PSI: Pneumonia Severity Index; CURB-65: confusion (abbreviated mental test score of $\leqslant 8$ ), urea $\geqslant 7 \mathrm{~mol} \cdot \mathrm{L}^{-1}$, respiratory rate $\geqslant 30$ breaths $\cdot \mathrm{min}^{-1}$, blood pressure $<90 \mathrm{mmHg}$ systolic or $<60 \mathrm{mmHg}$ diastolic, and age $\geqslant 65 \mathrm{yrs}$; MR: midregional; proADM: proadrenomedullin; CRP: C-reactive protein; PCT: procalcitonin; WBC: white blood cells; AUC: area under the curve. Dependent variable: mortality. Bold indicates statistically significant $\mathrm{p}$-values.

A Swiss group observed that a model including MR-proADM and PSI significantly increased the prediction accuracy over that of the PSI score alone for treatment failure [11], and 30-day mortality [13], whereas a German study found an improvement of prognostic power for the CRB-65 score after adding MR-proADM [12]. However, in the study by HuANG et al. [14], MR-proADM levels did not alter PSI-based risk assessment in most CAP patients, although this may be partly due to the high number of low-risk CAP patients $(60.6 \%)$ in their cohort. Recently, ALBRICH et al. [23] reported, in a cohort of CAP and non-CAP lower respiratory tract infection patients, that a risk score based on CURB-65 classes enhanced by MR-proADM cut-off ranges improved prognostic assessment of this clinical score, in terms of death of any cause, ICU admission or complications within 30 days after enrolment.

Our optimal cut-off for MR-proADM for predicting 30-day mortality was $1.066 \mathrm{nmol} \cdot \mathrm{L}^{-1}$. CHRIST-CRAIN et al. [11] proposed a cut-off for short-term mortality (mean 6.9 weeks) of $1.8 \mathrm{nmol} \cdot \mathrm{L}^{-1}$ and HuANG et al. [14] of $1.3 \mathrm{nmol} \cdot \mathrm{L}^{-1}$ for 30 -day mortality. This latter cut-off, which is higher than our value, was obtained from a lower-severity CAP cohort (60\% were PSI I-III), but with surprisingly higher short-term mortality $(6.4 \%)$ [14]. KRÜGER et al. [12] found a MR-proADM cut-off for shortand long-term mortality that was slightly lower than our value $\left(0.959 \mathrm{nmol} \cdot \mathrm{L}^{-1}\right)$, but severity was also lower in their cohort.

MR-proADM with PSI and CURB-65 scores separately distinguished mid- and long-term mortality from survival after CAP. Three studies found a correlation between long-term mortality and a high PSI score in the acute phase of CAP $[4,5$, 15]. GUERTLER et al. [15] observed that a model including male sex, COPD, neoplastic disease and peak MR-proADM levels was significantly better at discriminating 18-month survivors from nonsurvivors than PSI. In our ROC analysis, the 1-yr mortality AUC for PSI improved significantly after adding MR-proADM. In terms of short-term mortality, death in all but one of our patients who died after 30 days was due to causes other than the initial CAP, and the causes of death in our study were similar to those in other published studies [24, 25]. Four $(23.5 \%)$ patients died during the year after admission due to a new case of pneumonia.

We found only significant relationship between MR-proADM (and clinical scores) and short- and long-term mortality. The limited number of patients could be a reason for the lack of prognostic power of PCT in our study, which has been found in other larger series [6]. It is not yet clear whether a relationship between high MR-proADM levels and lower long-term survival is because a worse general condition is associated with lower immune function, thereby facilitating the spread of respiratory infections, or because a more severe infection exacerbates the underlying chronic diseases of the patients, or both [15].

With regards to the aetiological agents detected, two findings deserve comment. First, we cannot be sure that the viruses detected really played a pathogenetic role in our CAP patients, instead of representing possible prolonged viral shedding or even a coincidental upper airway infection [26]; however, the different viral yield obtained from patients and controls can help us to conclude that, at least in an important number of cases, viruses were a true pathogen in our CAP patients. Secondly, the relatively high rate of Pseudomonas aeruginosa in our population may be due to the inclusion of patients with bronchiectasis.

We found that PCT was the best biomarker, when compared with CRP and MR-proADM, for discriminating CAP involving typical bacteria (bacterial and mixed groups) from viral (and atypical) CAP. PCT levels in CAP due to M. pneumoniae and C. pneumoniae have been found to be low, although severe CAP 
TABLE 7 Aetiology of community-acquired pneumonia patients

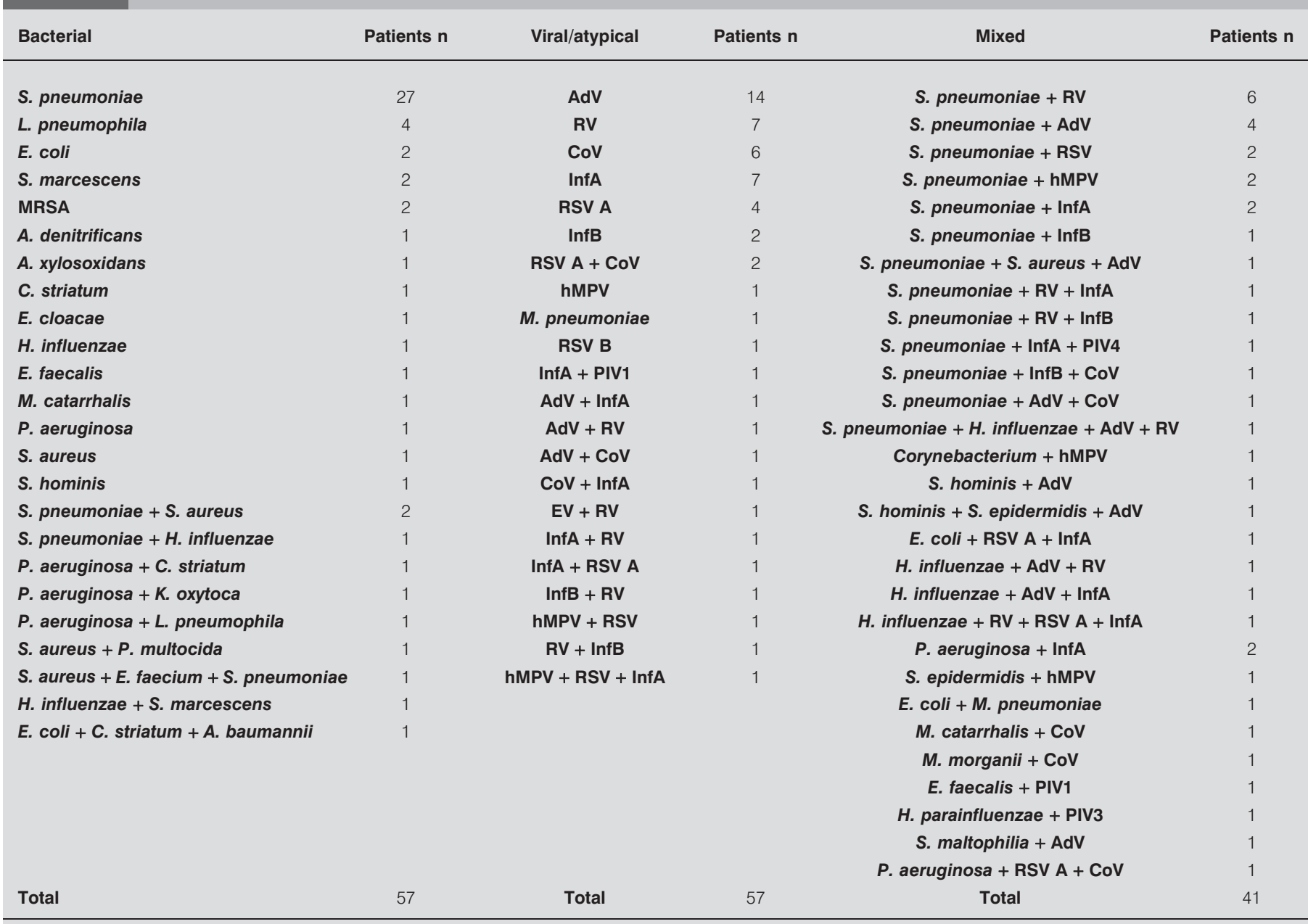

Out of 228 patients, aetiology was known in 155 (67.98\%) and unknown in 73 (32.02\%). S. pneumoniae: Streptococcus pneumoniae; L. pneumophila: Legionella pneumophila; E. coli: Escherichia coli; S. marcescens: Serratia marcescens; MRSA: methicillin-resistant Staphylococcus aureus; A. denitrificans: Alcaligenes denitrificans; A. xylosoxidans: Alcaligenes xylosoxidans; C. striatum: Corynebacterium striatum; E. cloacae: Enterobacter cloacae; H. influenzae: Haemophilus influenzae; E. faecalis Enterococcus faecalis; M. catarrhalis: Moraxella catarrhalis; P. aeruginosa: Pseudomonas aeruginosa; S. aureus: Staphylococcus aureus; S. hominis: Staphylococcus hominis: K. oxytoca: Klebsiella oxytoca; P. multocida; Pasteurella multocida: A. baumannii: Acinetobacter baumannii; AdV: adenovirus; RV: rhinovirus; CoV: coronavirus; Inf: influenza virus; hMPV: human metapneumovirus; RSV: respiratory syncytial virus; PIV: parainfluenza virus; M. pneumoniae: Mycoplasma pneumoniae; EV: enterovirus; S. epidermidis: Staphylococcus epidermidis; M. morgannii: Morganella morganii; H. parainfluenzae: Haemophilus parainfluenzae; S. maltophilia: Stenotrophomonas maltophilia.

due to L. pneumophila may show higher values [16]. In our study, plasma levels of MR-proADM were similar among patients infected with the three groups of microorganisms, and we found no association between MR-proADM and aetiology. This indicates that initial values of MR-proADM do not depend on the causes of CAP.

\section{TABLE 8 Levels of biomarkers in aetiological groups}

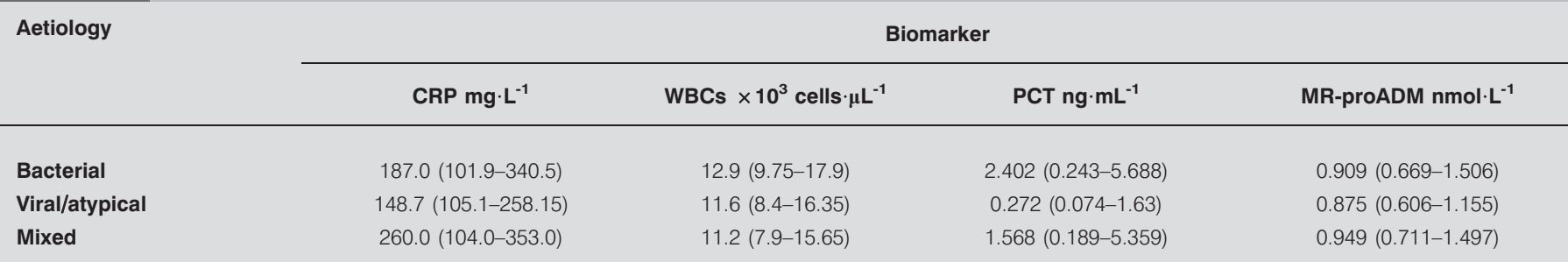

Data are presented as median (interquartile range). CRP: C-reactive protein; WBC: white blood cell; PCT: procalcitonin; MR: midregional; proADM: proadrenomedullin 
There are two clinical implications of our study. First, MRproADM is the best biomarker to predict short-term outcome in hospitalised CAP, whatever its aetiology. Secondly, it can be used to monitor the long-term follow-up of CAP patients discharged from hospital. Obviously, the final objective is to help to decrease mortality of CAP. These considerations are in line with a recent perspective article by EWIG and TORRES [27].

The limitations of our study merit consideration. The most important limitation is that this was a single-hospital study. Furthermore, we did not study biomarkers serially and their values may therefore change during the patient's course, thereby making it possible to better identify deterioration or improvement.

We conclude that plasma levels of MR-proADM, obtained within $6 \mathrm{~h}$ of arrival at the hospital, have considerable prognostic value, independently of the causal agent of CAP. Furthermore, our results confirm previous reports that MRproADM has a similar short-, mid- and long-term predictive power to that of the PSI and CURB-65 scores, has a good correlation with them and, when added to these clinical scores, improves their prognostic accuracy.

\section{SUPPORT STATEMENT}

The study was partially funded by a 2010 grant from the Aragon Respiratory Apparatus Society, the 2009 Brahms Prize from the José Luis Castaño Foundation for the Development of Clinical Biochemistry of the Spanish Society for Clinical Biochemistry and Molecular Pathology and Brahms Iberia SL. The funding organisations played no role in the study design, data collection and analysis, or manuscript approval. Brahms Iberia SL provided instrumentation and reagents for MR-proADM and, partially, PCT determinations. Sample and Assays Technologies performed free of charge the second PCR viral test in Hamburg, Germany.

\section{STATEMENT OF INTEREST}

Statements of interest for A.B. Lasierra and A. Torres, and for the study itself can be found at www.erj.ersjournals.com/site/misc/statements. xhtml

\section{ACKNOWLEDGEMENTS}

M.J. Revillo (Microbiology Dept, Hospital Universitario Miguel Servet, Zaragoza, Spain), M. Omeñaca (Virology Section, Hospital Universitario Miguel Servet) and C. Villuendas (Bacteriology Section, Hospital Universitario Miguel Servet) coordinated microbiological tasks. A.G. de Jalón (Biochemistry Dept, Hospital Universitario Miguel Servet) guided biomarker quantification. A.L. Simon (Pulmonology Dept, Hospital Universitario Miguel Servet) helped with data and nasopharyngeal aspirate collection. ED nurses obtained blood, sputum and urine samples, whereas ED physicians collaborated in initial diagnosis of patients.

\section{REFERENCES}

1 Fine MJ, Auble TE, Yealy DM, et al. A prediction rule to identify low-risk patients with community-acquired pneumonia. $N$ Engl J Med 1997; 336: 243-250.

2 Lim WS, van der Eerden MM, Laing R, et al. Defining community acquired pneumonia severity on presentation to hospital: an international derivation and validation study. Thorax 2003; 58: 377-382.

3 Capelastegui A, España PP, Quintana JM, et al. Validation of a predictive rule for the management of community-acquired pneumonia. Eur Respir J 2006; 27: 151-157.

4 Mortensen EM, Kapoor WN, Chang CC, et al. Assessment of mortality after long-term follow-up of patients with communityacquired pneumonia. Clin Infect Dis 2003; 37: 1617-1624.
5 Johnstone J, Eurich DT, Majumdar SR, et al. Long-term morbidity and mortality after hospitalization with community-acquired pneumonia: a population-based cohort study. Medicine (Baltimore) 2008; 87: 329-334.

6 Niederman MS. Biological markers to determine eligibility in trials for community-acquired pneumonia: a focus on procalcitonin. Clin Infect Dis 2008; 47: Suppl. 3, S127-S132.

7 Krüger S, Ewig S, Papassotiriou J, et al. Inflammatory parameters predict etiologic patterns but do not allow for individual prediction of etiology in patients with CAP - results from the German competence network CAPNETZ. Respir Res 2009; 10: 65.

8 Christ-Crain M, Stolz D, Bingisser R, et al. Procalcitonin guidance of antibiotic therapy in community-acquired pneumonia: a randomized trial. Am J Respir Crit Care Med 2006; 174: 84-93.

9 Menéndez R, Martínez R, Reyes S, et al. Biomarkers improve mortality prediction by prognostic scales in community-acquired pneumonia. Thorax 2009; 64: 587-591.

10 Krüger S, Ewig S, Marre R, et al. Procalcitonin predicts patients at low risk of death from community-acquired pneumonia across all CRB-65 classes. Eur Resp J 2008; 31: 349-355.

11 Christ-Crain M, Morgenthaler NG, Stolz D, et al. Pro-adrenomedullin to predict severity and outcome in community-acquired pneumonia. [ISRCTNO4176397]. Crit Care 2006; 10: R96.

12 Krüger S, Ewig S, Giersdorf $S$, et al. Cardiovascular and inflammatory biomarkers to predict short- and long-term survival in community-acquired pneumonia. Results from the German competence network CAPNETZ. Am J Respir Crit Care Med 2010; 182: $1426-1434$.

13 Schuetz P, Wolbers M, Christ-Crain M, et al. Prohormones for prediction of adverse medical outcome in community-acquired pneumonia and lower respiratory tract infections. Crit Care 2010; 14: R106.

14 Huang DT, Angus DC, Kellum JA, et al. Midregional proadrenomedullin as a prognostic tool in community-acquired pneumonia. Chest 2009; 136: 823-831.

15 Guertler C, Wirz B, Christ-Crain M, et al. Inflammatory response predict long-term mortality risk in community-acquired pneumonia. Eur Respir J 2011; 37: 1439-1446.

16 Haeuptle J, Zaborsky, Fiumefreddo R., et al. Prognostic value of procalcitonin in Legionella pneumonia. Eur J Clin Microbiol Infect Dis 2009; 28: 55-60.

17 Alfageme I, Aspa J, Bello S, et al. Normativas para el diagnostico y el tratamiento de la neumonía adquirida en la comunidad. Sociedad Española de Neumología y Cirugía Torácica (SEPAR) [Guidelines for the diagnosis and management of communityacquired pneumonia. Spanish Society of Pulmonology and Thoracic Surgery (SEPAR)]. Arch Bronconeumol 2005; 41: 272-289.

18 Morgenthaler NG, Struck J, Alonso C, et al. Measurement of midregional proadrenomedullin in plasma with an immunoluminometric assay. Clin Chem 2005; 51: 1823-1829.

19 Coiras MT, Aguilar JC, Garcia ML, et al. Simultaneous detection of fourteen respiratory viruses in clinical specimens by two multiplex reverse transcription nested-PCR assays. J Med Virol 2004; 72: 484-495.

20 Sample and Assay Technologies. ResPlex II handbook. Hamburg, Qiagen, 2010.

21 Christ-Crain M, Morgenthaler NG, Struck J, et al. Mid-regional pro-adrenomedullin as a prognostic marker in sepsis: an observational study. Crit Care 2005; 9: R816-R824.

22 Guignant C, Voirin N, Venet F, et al. Assessment of pro-vasopressin and pro-adrenomedullin as predictors of 28-day mortality in septic shock patients. Intensive Care Med 2009; 35: 1859-1867.

23 Albrich WC, Dusemund F, Ruegger K, et al. Enhancement of CURB65 score with proadrenomedullin (CURB65-A) for outcome prediction in lower respiratory tract infections: derivation of a clinical algorithm. BMC Infect Dis 2011; 11: 112.

24 Mortensen EM, Coley CM, Singer DE, et al. Causes of death for patients with community-acquired pneumonia. Results from the 
Pneumonia Patient Outcomes Research Team Cohort Study. Arch Intern Med 2002; 162: 1059-1064.

25 Yende S, D'Angelo G, Kellum JA, et al. Inflammatory markers at hospital discharge predict subsequent mortality after pneumonia and sepsis. Am J Respir Crit Care Med 2008; 177: 1242-1247.
26 Ruuskanen O, Lathi E, Jennings LC, et al. Viral pneumonia. Lancet 2011; 377: 1264-1275.

27 Ewig S, Torres A. Community-acquired pneumonia as an emergency: time for an aggressive intervention to lower mortality. Eur Respir J 2011; 38: 253-260. 